\title{
To boldly go
}

\author{
China is investing in big astronomy and astrophysics projects, but is still debating the way forward in \\ experimental particle physics.
}

Last year, China made the headlines with the launch of the world's first quantum communications satellite, the completion of the Five-hundred-meter Aperture Spherical radio Telescope (FAST) and an ambitious fast-paced space program. Such achievements may be taken to indicate that China's spectacular scientific growth has expanded and branched into astronomy and particle physics, where it will soon take the lead.

These developments have to be placed into context. Lacking home facilities, Chinese astronomers and particle physicists have long been going abroad to use telescopes, observatories or particle accelerators. Historically, investment in fundamental science has not been a priority for the Chinese government. And, although facilities such as the upcoming reactor neutrino experiment at the Jiangmen Underground Neutrino Observatory, the dark matter detector PandaX-II at the Jinping Underground Laboratory and the Dark Matter Particle Explorer satellite are very welcome developments, an even bigger project has prompted an important debate within the Chinese scientific community.

Among the design proposals for the successor to the Large Hadron Collider is the Circular Electron Positron Collider (CEPC), a 50-100-kilometre-long accelerator ring that could cost 40 billion Chinese yuan (US\$5.7 billion). This project dwarfs even FAST, with

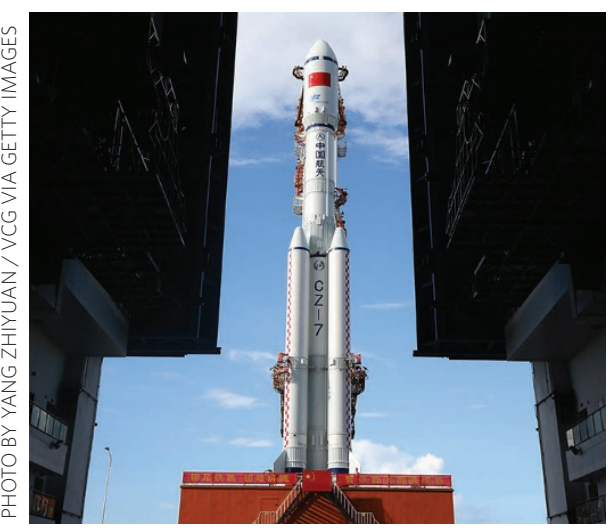

The Long March 7 carrier rocket ready for launch.

its 500-metre dish and 1.2 billion Chinese yuan budget. And it may be followed by a super proton-proton collider (SPPC), raising the budget to a little less than 100 billion yuan. These cost estimates are debatable (http://go.nature.com/2hvxNiq), but the order of magnitude is likely correct and, even by the standards of large-scale scientific projects, eye-wateringly expensive.

Last autumn, physics Nobel laureate Chen-Ning Yang vocally opposed the construction of CEPC-SPPC, as well as Chinese colliders in general, suggesting such an endeavour would be financially ill-advised. Yang drew an analogy with the unfortunate end of the Superconducting Super Collider project - the US particle collider whose construction was abandoned in 1993 after two billion dollars had been spent. And he questioned the wisdom of investing in what he believes is a similarly risky enterprise, arguing that the money should be put to better use for other science projects and to improve people's lives (http://go.nature.com/2hNL1qd).

Overt opposition to the project from a leading particle physicist was both unexpected and hard to dismiss. But Yifang Wang, the director of the Chinese Academy of Sciences Institute of High Energy Physics, which is leading the CEPC-SPPC project, immediately replied to Yang's points. And he was joined by Fields medallist Shing-Tung Yau, who has long been an advocate for the construction of Chinese colliders.

Yang certainly raises some fair questions. What are the actual costs? Are they worth it? Why not invest in other areas of science that have a clearer impact on society? And will a larger collider be able to significantly advance our fundamental understanding? Wang, Yau and other supporters make compelling arguments. This debate might sound familiar in the West, but this is the first time such a discussion has been brought out into the open in China. It is certainly worth involving the general public, because whereas space research is easy to sell, the merits and attraction of exploring the subatomic world needs more promotion.

\section{A new launch}

\section{This month we officially welcome our new sister journal, Nature Astronomy.}

A few new titles have recently joined the Nature family, publishing their first issues this month. One in particular is close to the hearts of the Nature Physics editors - and not just in terms of its topic. The newly launched Nature Astronomy is led by one of the first editors of Nature Physics, May Chiao. May was part of the team that launched Nature Physics, and stayed with us for over ten years. During that time she handled an eclectic editorial brief that included, among other things, astrophysics. Her flair for a pithy title has certainly coloured our pages. And as sad as we are to see her leave, we wish her and all the Nature Astronomy editors the best.

Nature has a long tradition of publishing astronomy - unsurprising given that its founder and first editor was a British astronomer, Sir Norman Lockyer. The astronomy content has been complemented by more astrophysics and planetary science in the pages of Nature Physics and Nature Geoscience. Now, Nature Astronomy aims to bring astronomers, astrophysicists and planetary scientists together in one place, and foster the interaction among these communities.

We are keen to play our role in promoting this dialogue and to engage more with the astrophysics community. We are looking forward to joint editorial projects with Nature Astronomy (watch this space!) and hope that together we can offer a more diverse outlet for the astrophysics community. Meanwhile, do check out our friends online at www.nature.com/natastron and on Twitter@NatureAstronomy. 